\title{
Crystal Growth of Pure and Impurity-Doped Lead Chloride Single Crystals from the Melt
}

\author{
B. WILLEMSEN \\ Department of Solid State Chemistry, University of Utrecht, The Netherlands
}

Received March 18, 1971

\begin{abstract}
An account is given of the purification and the crystal growing of lead chloride. Difficulties observed are blackening of the material and the formation of oxides. It is evident that oxygen should be avoided. The influence of water vapour is studied with mass spectroscopy, crystal microbalance measurements, and microscopical observations.

Water vapour reacts with lead chloride giving lead (hydr)-oxychlorides and hydrochloric acid, but is not responsible for the blackening. On the surface of freshly cleaved crystals, which are exposed to water vapour rod-shaped hydrolysis products, are observed lying in the direction of the crystallographic $a$ axis.

The blackening is not a thermal decomposition of lead chloride itself, but is attributed to organic contaminations.
\end{abstract}

\section{Introduction}

Lead chloride has been the subject of many investigations in our laboratory. In the past, the ionic conductivity was measured $(1,2)$, the photolysis investigated (3), and electron spin resonance measurements performed on lead chloride doped with europium (II) (4) and gadolinium (III) (5). For all these investigations, single crystals of good quality had to be prepared. In this article, the preparation of both doped and undoped crystals will be described. Special attention will be paid to the phenomena of blackening and hydrolysis, which may occur during the preparation process.

\section{Purification of the Starting Material}

The starting material for the preparation of lead chloride was reagent-grade $\mathrm{PbCl}_{2}$ (J. T. Baker Co). The impurity content as given by the supplier is shown in Table I, together with two additional mass-spectrometric determinations of our own.

Continued purification by recrystallization from an aqueous solution is not required, because of the low impurity content of the starting material. Efforts to prepare lead chloride single crystals of high quality from this material have failed. All preparations gave crystals contaminated with lead oxides. The resulting crystals have a brown colour. Moreover, they show black spots.

It was observed, that the lead chloride melt was intense black, which resulted in the aforementioned black spots in the grown crystals. The black colour of the melt and the black spots are ascribed to an excess of lead, as we shall discuss below. Therefore, we looked for a process that removes the lead oxides as well as the excess of lead.

Two kinds of processes were compared:

1. Sublimation or distillation methods,

2. Zone-refining method.

TABLE I

IMPURITY CONTENT IN THE STARTING MATERIAL (WT\%)

\begin{tabular}{llc}
\hline \multicolumn{1}{c}{ Given by the supplier } & $\begin{array}{c}\text { Mass spectrometric } \\
\text { determinations }\end{array}$ \\
\hline Unsoluble & 0.006 & \\
Not precipitated by $\mathrm{H}_{2} \mathrm{~S}$ & 0.010 & \\
$\mathrm{Cu}$ & 0.001 & \\
$\mathrm{Fe}$ & 0.0001 & \\
$\mathrm{H}_{2} \mathrm{O}$ & & 0.03 \\
Organic material & & detectable \\
\hline
\end{tabular}


Sublimation appears to be a very slow process for the production of large amounts of lead chloride. However, very pure crystals can be obtained (I). Vacuum distillation does not give a colourless product, at least not if a simple distillation apparatus is used. This is probably due to entrainment of the boiling liquid.

For production of large amounts of very pure lead chloride, the zone-refining method proved to be most suitable.

As stated above, the melt becomes intensely black. It contains black particles, which are suspended in the melt and cannot be transported by the molten zone. Probably an excess of lead is present. Therefore, we tried to clear the melt by a chlorine atmosphere. Under this condition the melt becomes clear-yellow, and the lead oxides, mainly lead (II) oxide, can be eliminated by a conventional zonerefining process.

There exist other possibilities to eliminate the excess of lead. The first is melting under an oxygen atmosphere. Presumably, the excess of lead is then converted to lead oxides or lead oxychlorides. After this the oxygen atmosphere was removed and replaced by nitrogen. A piece of solid sublimated ammonium chloride was added to the melt in order to decrease the lead oxide contents. The remaining oxides could then be removed by zone-refining.

The second method, preferred by us, is direct refining of the starting material under a stream of nitrogen saturated with carbon tetrachloride. This atmosphere has chlorinating properties and diminishes both the excess of lead and the oxide content. As soon as the melt and the solid material is transparent, the process is continued under nitrogen only. No blackening occurs during the further continuation of the zone-refining process. Not all of the oxides are converted, since the end of the batch is brownishly stained. The total number of zone-passes amounts to twenty to forty, depending on the further application of the purified material.

\section{Crystal Growth}

Two techniques were at our disposal; these are the horizontal zone and the vertical lowering technique according to Bridgman. The horizontal zone does not yield perfect crystals of large size. Unless small crystals are acceptable, the Bridgman technique was preferred.

The furnaces used are of the two-section type. The best results are obtained, when ampullae of supremax glass are used, with a diameter only a little smaller than the inner diameter of the furnace. Typical
TABLE II

\begin{tabular}{|c|c|c|}
\hline \multicolumn{3}{|c|}{ 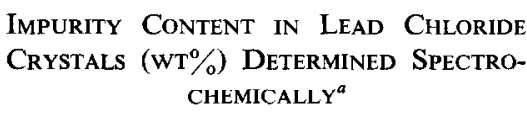 } \\
\hline \multicolumn{2}{|c|}{ Undoped } & \multirow{2}{*}{ 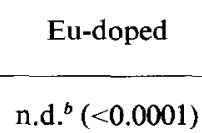 } \\
\hline $\mathrm{Si}$ & 0.002 & \\
\hline $\mathrm{Mg}$ & 0.0001 & 0.0002 \\
\hline $\mathrm{Fe}$ & n.d. ${ }^{b}$ & n.d. ${ }^{b}(<0.0002)$ \\
\hline $\mathrm{Al}$ & 0.0008 & 0.00005 \\
\hline $\mathrm{Cu}$ & n.d. ${ }^{b}$ & 0.00005 \\
\hline $\mathrm{Eu}$ & & 0.1 \\
\hline
\end{tabular}

\footnotetext{
a Thanks are due to The Philips Research Laboratories at Eindhoven for these analyses.

${ }^{b}$ n.d. = not detected.
}

dimensions are an inner diameter of $21 \mathrm{~mm}$ of the furnace and an outer diameter of $18 \mathrm{~mm}$ of the ampullae.

The purified lead chloride is melted and poured into the ampullae. These are zone-refined again, in order to eliminate possible new impurities. After this one quarter of the content is discarded. Finally, the ampullae are sealed under vacuum and lowered in the furnace.

For the preparation of doped lead chloride, the procedure described above is somewhat modified. The oxides of gadolinium or europium are dissolved in hydrochloric acid. An excess of ammonium chloride is added to the solution. This solution is then evaporated to dryness and the remainder dried at a temperature of $150^{\circ} \mathrm{C}$. The mixture and the purified lead chloride are transferred to a fritted glass-filter. The ammonium chloride is slowly evaporated under vacuum, then temperature is raised, until the lead chloride is molten. The doped lead chloride is received in a container or directly in a crystallization ampulla.

Optically transparent crystals are obtained, doped as well as undoped, with dimensions of a few centimeters long. Incidentally, larger crystals are grown. Table II gives the spectrochemically determined impurity contents. The oxide content is determined by activation analysis to be less than ten parts per million.*

From ESR measurements it follows that europium is built in with a valency of two; optically it can be shown that a trace of europium with a valency three is also present. Gadolinium is built in with a valency

\footnotetext{
* R. C. N. Petten, private communication.
} 
of three, this is verified by measuring the ionic conductivity (12).

\section{Hydrolysis of Lead Chloride}

As mentioned above, lead chloride shows a blackening at elevated temperatures. This does not seem to be a simple thermal decomposition of the lead chloride, because no thermal blackening occurs if purified lead chloride is not contaminated afterwards, e.g., by exposing it to air. It is obvious that the influence of moisture and oxygen should be investigated.

It is a well-known fact that at high temperatures oxygen causes the formation of oxides and oxychlorides (6).

The action of water can be shown qualitatively by passing a stream of wet nitrogen over purified lead chloride at a temperature of $400^{\circ} \mathrm{C}$. In the gas stream, $\mathrm{HCl}$ can be detected and the surface of the lead chloride becomes brown. With dry nitrogen no $\mathrm{HCl}$ is observed.

The mechanism of hydrolysis at room-temperature is investigated by the quarz crystal micro-balance technique (7). We made a fresh lead chloride layer by the reaction of a lead layer with chlorine. After the removal of chlorine we introduced water vapour. It appears that directly after introducing water vapour the mass of the layer decreases, followed by an increase (Fig. 1). This experiment can be interpreted as the substitution of $\mathrm{Cl}$ by $\mathrm{O}$ or $\mathrm{OH}$, forming hydroxy- or oxychlorides, followed by continued water adsorbtion.

Also the influence of water vapour on the surface of lead chloride crystals was studied. The surface of freshly prepared and cleaved crystals shows after a few days storage in water vapour ( $18 \mathrm{~mm}$ pressure)

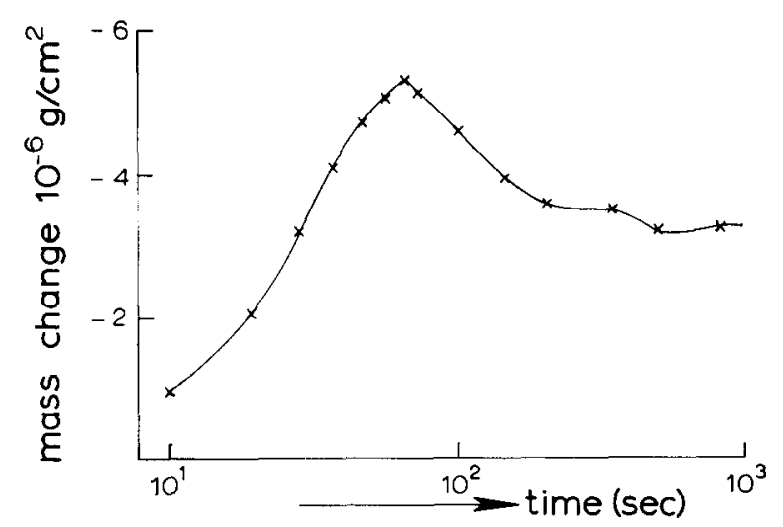

FIG. 1. The change of mass of a $\mathrm{PbCl}_{2}$ layer exposed to water vapour $(16 \mathrm{~mm})$ as a function of time. at room temperature characteristic rods on the cleavage plane. These rods lie in the direction of the $a$ axis. After storage during a week in water vapour, the rods increase to larger structures (Fig. 2).

The rods as well as the larger structures were investigated by an electron microprobe scanning analyser. From a line scan made across the structures it appears that the rods as well as the structures show a deficiency of chlorine (Fig. 3). We looked also for oxygen on the surface, but without result. The analyser is rather insensible to oxygen. This was confirmed with an experiment on pure lead oxide. Moreover, the electron beam destroys the surface somewhat, so that there is no evidence that the structures do not contain oxygen. Finally, the occurrence of carbon on the surface is investigated. Carbon was detected, but there is no correlation with the visible surface structures.

When crystals with the surface structures are heated in vacuum, the structures disappear at temperatures above $300^{\circ} \mathrm{C}$. The surface has a uniform brownish tarnish. Presumably, the structures are composed of lead hydroxychloride, which is dehydroxylated by heating, leaving a lead oxide layer:

$2 \mathrm{~Pb}(\mathrm{OH}) \mathrm{Cl} \stackrel{177-246^{\circ} \mathrm{C}}{\longrightarrow} \mathrm{PbO}(\mathrm{s})+\mathrm{PbCl}_{2}(\mathrm{~s})+\mathrm{H}_{2} \mathrm{O}(\mathrm{g})$.

We may conclude that water vapour gives hydrolysis on the surface introducing lead oxide contamination. The hydrolysis is not responsible for an excess of lead at elevated temperatures.

\section{Thermal Blackening of Lead Chloride}

When heating lead chloride crystals in vacuum blackening occurs incidentally, mostly on edges or irregular fractures. When the outer layers arc evaporated, lead-rich particles remain situated on the edges (Fig. 4). It is not possible to cause blackening by scratching the surface.

When heating commercial lead chloride powder, local black spots appear and the powder becomes gray at about $300^{\circ} \mathrm{C}$. Powdered and purified lead chloride does not show such black spots, but becomes less white.

Obviously, moisture and oxygen must be avoided, but it is not clear why the blackening occurs. Simple mass spectrometer experiments give some information on the partial pressures of the released gasses at elevated temperatures. Samples of commercially available lead chloride and purified lead chloride were compared at different temperatures. 


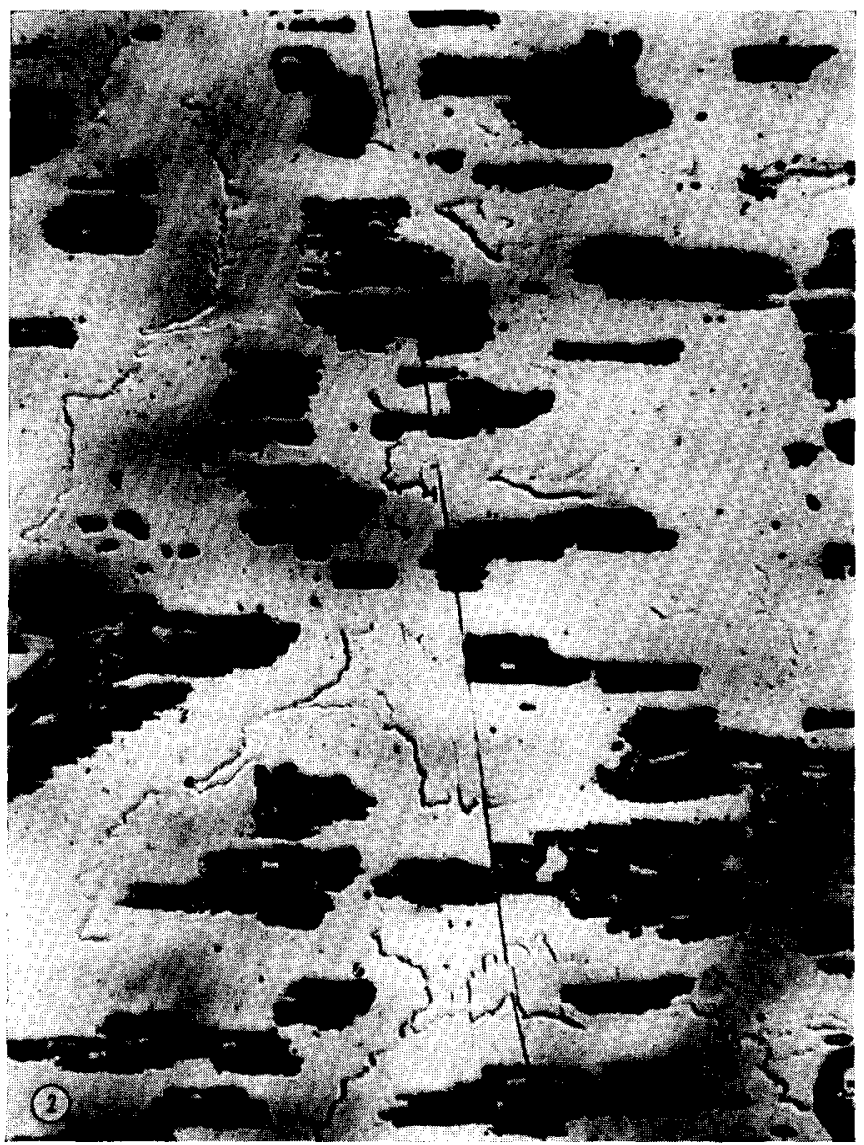

Fig. 2. (001) surface of cleaved lead chloride crystals after eight days storage in water vapour at $20^{\circ} \mathrm{C}$. Microscope: Leitz with transmitted light. Magn. $\times 300$.

When heating the samples, mainly three kinds of gasses were detected, viz., water, halogen, and carbon dioxide.

Samples of powdered commercially available lead chloride are thoroughly dried in vacuum, meanwhile the partial pressure of water and the temperature is measured continuously. It appears very difficult to eliminate all the water from the material, since at $300^{\circ} \mathrm{C}$ there is still a partial pressure of water considerably larger than the background of the mass spectrometer. This is the case even after $\mathbf{5 0}$ hours of drying with slowly rising temperature. Presumably, this corresponds to a dehydroxylation of lead hydroxy chloride $(8)$ :

$$
2 \mathrm{~Pb}(\mathrm{OH}) \mathrm{Cl} \stackrel{177-246^{\circ} \mathrm{C}}{\longrightarrow} \mathrm{PbO}(\mathrm{s})+\mathrm{PbCl}_{2}(\mathrm{~s})+\mathrm{H}_{2} \mathrm{O}(\mathrm{g}) .
$$

The total quantity of water desorbed is estimated (Table I).

In the temperature region between $200^{\circ} \mathrm{C}$ and $250^{\circ} \mathrm{C}$ halogen becomes detectable. It is not possible to conclude to $\mathrm{HCl}$ or $\mathrm{Cl}_{2}$, because in the apparatus $\mathrm{Cl}_{2}$ is converted into $\mathrm{HCl}$. There is a striking difference between the halogen desorption of powdered samples of the starting material and of the purified material (Fig. 5). This commercial powder shows a larger halogen pressure at the same temperature. This difference cannot be attributed to the size of the lead chloride grains, because the grain size of the commercial powder and the grain size of the purified powders is in the same order of magnitude. Microscopical observations show that the grains of the pure powder presumably have a much larger surface. After storage of 24 hours in moist air a powder of the purified material shows only a slight increase in halogen desorbtion. So there is no direct connection between halogen desorbtion and moisture. From the temperature dependence of the partial pressure of halogen, it is possible to estimate "activation energies" for the release of halogen, which show a rather large variation, viz., between 15 and $30 \mathrm{kCal} / \mathrm{mole}$. We may conclude therefore 


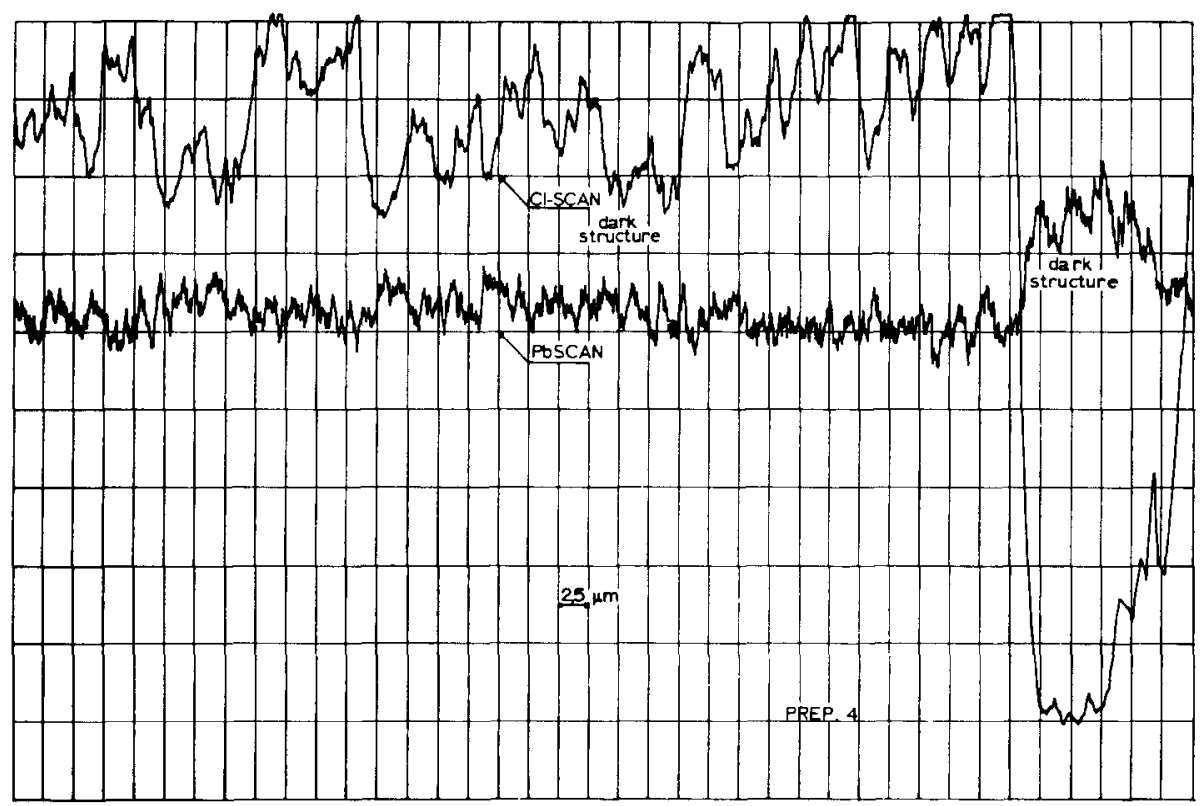

Fig. 3. Line scan across surface detail of Fig. 2 with an electron microprobe scanning analyser. $\mathrm{Pb}$ and $\mathrm{Cl}$ are simultaneously scanned.

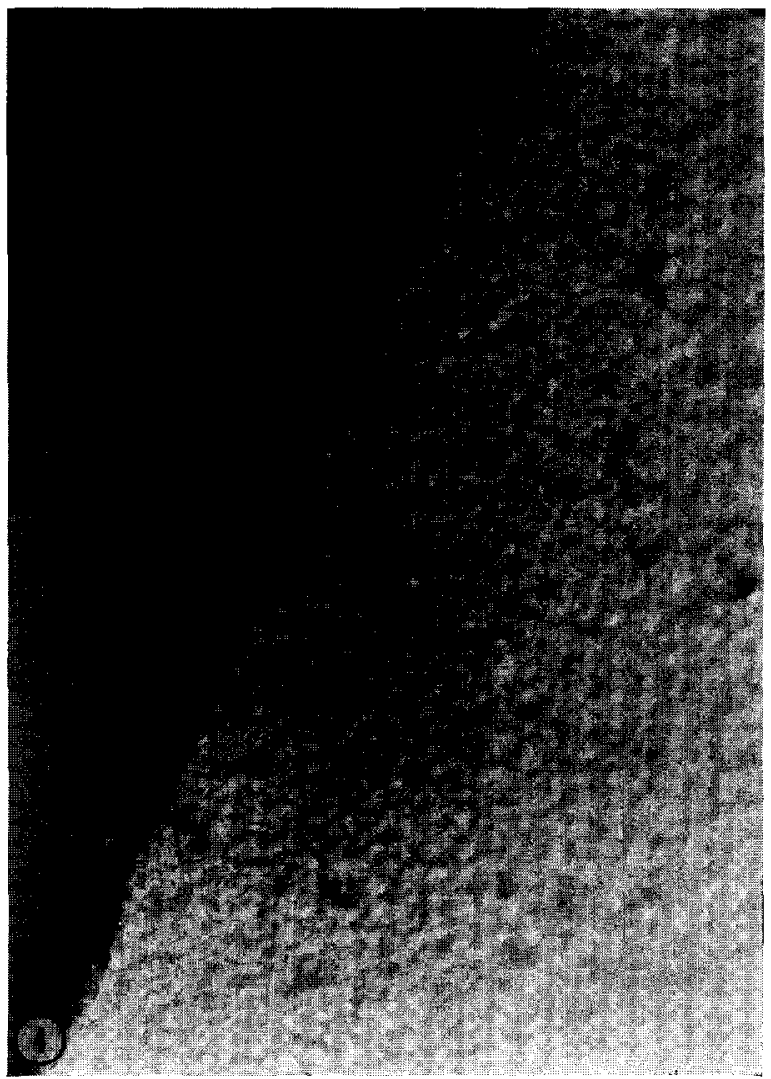

Fig. 4. Edge of a lead chloride crystal, heated in vacuum at $350^{\circ} \mathrm{C}$, with black particles on the edge. Microscope: Leitz with reflecting light. Magn, $\times 300$. 


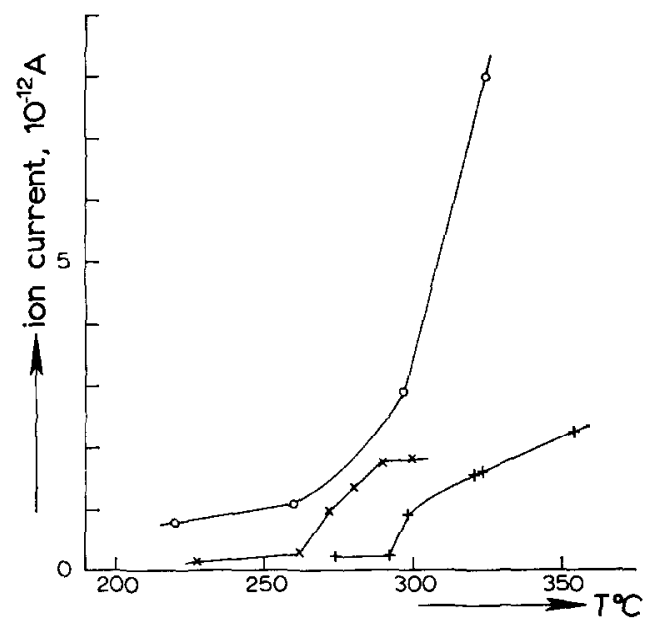

Fig. 5. Ion current in the mass spectrometer for ${ }^{35} \mathrm{Cl}$ plotted versus temperature of lead chloride.

000 Lead chloride (Baker Co.)

+++ Purified lead chloride (powder)

$x \times x \quad$ Purified lead chloride (powder) after storage in water vapour.

that there is more than one process by which halogen is generated.

In addition to halogen, we detected in all cases carbon dioxide at elevated temperatures. The total amount of carbon dioxide was roughly forty times larger for the commercial powders than for the purified powders. This indicates the presence of organic contamination in the commercial powder. There is a rough analogy between the time and temperature-dependence of the partial pressures of halogen and carbon dioxide.

These experiments do not indicate a direct relation between water vapour and blackening. The blackening appears to be strictly local. Possibly, it corresponds to a reduction of the lead chloride. It seemed interesting to investigate the behavior of lead chloride in a reducing atmosphere, e.g., hydrogen, at elevated temperatures. Molten transparent lead chloride blackens in a few seconds in a hydrogen atmosphere. If a single crystal is heated for half an hour in hydrogen with a pressure of 1 atmosphere and a temperature of $300^{\circ} \mathrm{C}$, it blackens. The optical density for visible light increases with $\Delta D=0.006$. The dark surface layer is uniform, but surface irregularities are black-decorated.

Returning to the powder experiments, there are two reasons for halogen loss at elevated temperatures:

1. Reaction of the lead chloride with water vapour giving $\mathrm{HCl}$ and $\mathrm{PbO}$,
2. Reduction of $\mathrm{PbCl}_{2}$, possibly by organic contaminations.

The second process is responsible for the extra halogen loss and blackening of the commercial powder.

\section{Discussion}

The mass spectrometer experiments give us direct evidence for a large influence of storage in watcr vapour. We assume that the purified material reacts fast with water vapour (Fig. 1), i.e., saturation is quickly reached on the surface, while water inclusions do not exist. So there is only a small difference between a purified lead chloride powder exposed to water vapour and a powder not exposed, because, in both cases, it has already reacted with moisture during the handling of the powder. The halogen loss is caused only by a residue of water. The difference with the commercial powder must bc attributed to water inclusions and organic contamination. In addition, this shows the blackening mechanism.

The experiments concerning the hydrolysis and the blackening of lead chloride lead us to the conclusion that hydrolysis and blackening must be interpreted as different processes. Either process will cause halogen loss at elevated temperatures:

$$
\mathrm{PbCl}_{2}(\mathrm{~s})+\mathrm{H}_{2} \mathrm{O}(\mathrm{g}) \underset{\mathrm{T}>250^{\circ} \mathrm{C}}{\longrightarrow} \mathrm{PbO}(\mathrm{s})+2 \mathrm{HCl}(\mathrm{g}) \text {. }
$$

The halogen loss and blackening is not explicable as thermal decomposition of the lead chloride, because the purified material shows no blackening by heating or melting under nonequilibrium circumstances. From thermodynamical data, the equilibrium chlorine pressure of $\mathrm{PbCl}_{2}$ is calculated to be of the order of $10^{-20}$ atm at $400^{\circ} \mathrm{C}(1)$.

The blackening in an inert atmosphere appears to be connected with a reduction of the lead chloride as shown by the reaction with hydrogen. We suppose that organic contaminations on the chloride surface are oxidized giving $\mathrm{H}^{+}$ions causing desorption of $\mathrm{HCl}$. The lead chloride can be easily reduced because of the low activation energy of the migration of the $\mathrm{Cl}^{-}$vacancies. The mass spectrometric investigations show that in the commercial starting material, some organic contaminations must be present. This causes extra blackening and halogen loss. Moreover, careful drying of this powder has no effect on the blackening.

The growth of rods on the crystal surfaces is not clear, but we may assume that these rods consist of a 
coherent layer of hydrolysis products. The rods References seem to grow at preferential regions.

\section{Acknowledgments}

The author would like to thank Professor J. H. van Santen and Professor G. Blasse for encouraging this work and for many stimulating discussions. The author is grateful to Mr. D. Schalkoort, Metal Research Laboratory, Technical University, Delft for providing electron microprobe scanning analyser facilities and to H. J. van Ark MSc. for putting a mass spectrometer at his disposal. The skilful assistance of $\mathrm{Mr}$. G. J. Dirksen is also gratefully acknowledged.
1. K. J. De Vries and J. H. van Santen, Physica 29, 482, (1963).

2. K. J. DE VRits, Thesis, University of Utrechl, The Netherlands, 1967.

3. J. F. Verwey, J. Phys. Chem. Solids 27, 468 (1967).

4. Q. H. F. VReHen and J. Volger, Physica 31, 845 (1965).

5. H. C. W. Beijerinck and B. Willemsen, Physica 47,515 (1970).

6. J. N. MeLloR, "Comprehensive Treatise on Inorganic and Theoretical Chemistry," Vol. VII, Longmans, London, 1927.

7. J. M. L. Kerbusch, to be published.

8. P. Ramamurthy, E. A. Sicco,M. Badri, Can.J. Chem.48, 2617 (1970). 